\title{
Structure and functional dynamics characterization of the ion channel of the human respiratory syncytial virus (hRSV) small hydrophobic protein (SH) transmembrane domain by combining molecular dynamics with excited normal modes
}

\author{
Gabriela C. Araujo ${ }^{1}$ Ricardo H. T. Silva ${ }^{2}$ - Luis P. B. Scott ${ }^{2} \cdot$ Alexandre S. Araujo $^{1}$ • \\ Fatima P. Souza ${ }^{1} \cdot$ Ronaldo Junio de Oliveira ${ }^{3}$
}

Received: 29 September 2015 / Accepted: 13 October 2016 / Published online: 5 November 2016

(C) Springer-Verlag Berlin Heidelberg 2016

\begin{abstract}
The human respiratory syncytial virus (hRSV) is the major cause of lower respiratory tract infection in children and elderly people worldwide. Its genome encodes 11 proteins including SH protein, whose functions are not well known. Studies show that SH protein increases RSV virulence degree and permeability to small compounds, suggesting it is involved in the formation of ion channels. The knowledge of SH structure and function is fundamental for a better understanding of its infection mechanism. The aim of this study was to model, characterize, and analyze the structural behavior of SH protein in the phospholipids bilayer environment. Molecular modeling of SH pentameric structure was performed, followed by traditional molecular dynamics (MD) simulations of the protein immersed in the lipid bilayer. Molecular dynamics with excited normal modes (MDeNM) was applied in the resulting system in order to investigate long time scale pore dynamics. MD simulations support that SH protein is stable in its pentameric form.
\end{abstract}

Electronic supplementary material The online version of this article (doi:10.1007/s00894-016-3150-6) contains supplementary material, which is available to authorized users.

Fatima P. Souza

fatyssouza@gmail.com

1 Laboratório Multiusuário de Inovação Biomolecular, Departamento de Física, Instituto de Biociências, Letras e Ciências Exatas, Universidade Estadual Paulista "Júlio de Mesquita Filho", São José do Rio Preto, SP, Brazil

2 Laboratório de Biologia Computacional e Bioinformática, Universidade Federal do ABC, Santo André, SP, Brazil

3 Laboratório de Física Teórica, Departamento de Física, Instituto de Ciências Exatas, Naturais e Educação, Universidade Federal do Triângulo Mineiro, Uberaba, MG, Brazil
Simulations also showed the presence of water molecules within the bilayer by density distribution, thus confirming that $\mathrm{SH}$ protein is a viroporin. This water transport was also observed in MDeNM studies with histidine residues of five chains (His22 and His51), playing a key role in pore permeability. The combination of traditional MD and MDeNM was a very efficient protocol to investigate functional conformational changes of transmembrane proteins that act as molecular channels. This protocol can support future investigations of drug candidates by acting on SH protein to inhibit viral infection.

Keywords Excited normal modes $\cdot$ Molecular dynamics · $\mathrm{RSV} \cdot \mathrm{SH}$ protein $\cdot$ Viroporin

\section{Introduction}

Human respiratory syncytial virus (hRSV), an enveloped virus belonging to the genus Pneumovirus within the family Paramyxoviridae, is a major cause of lower respiratory tract infections in infants, elderly, and immunocompromised populations worldwide [1,2]. Most children have been infected at least once by hRSV before they reach 2 years old [3]. About 66,000-199,000 children die every year due to complications caused by hRSV infection [2].

$\mathrm{hRSV}$ is a negative-stranded RNA virus that expresses 11 known proteins from ten genes. The envelope of hRSV contains two surface glycoproteins involved in attachment and entry: G (attachment) and F (fusion) glycoproteins, which compose the major viral determinants [4]. The small hydrophobic $(\mathrm{SH})$ protein is an additional glycoprotein encoded by $\mathrm{hRSV}$ and a subset of paramixoviruses, including members of Pneumovirus. The specific function of SH in viral infections is not well understood due to experimental difficulties [5]; 
therefore, in this manuscript, the SH protein's mode of action was explored from a theoretical point of view.

A type II integral transmembrane glycoprotein, hRSV SH, is composed of 64 amino acids for hRSV type A, and 65 amino acids for type B [6]. SH exists in four different forms during infection, which vary according to its glycosylation level [7]: two non-glycosylated forms, a full length $7.5 \mathrm{KDa}$ form $\left(\mathrm{SH}_{0}\right)$ and a truncated 4.5 KDa species $\left(\mathrm{SH}_{\mathrm{t}}\right)$; an N-linked glycosylated form $(\mathrm{SHg})$, and a polylactosaminoglycan-modified form $\left(\mathrm{SH}_{\mathrm{p}}\right)$. All of them, except $\mathrm{SH}_{\mathrm{t}}$, are incorporated in the infected cells surface, and $\mathrm{SH}_{0}$ seems to be the most abundant form [8]. In our studies, the full-length form of $\mathrm{SH}_{0}$ was used.

SH protein significance for hRSV replicative cycle is unknown; however, it is known that SH is essential for an effective infection in two animal models: mice and chimpanzees [9, 10]. Some studies suggest that $\mathrm{SH}$ protein plays a role in a virus-mediated cell fusion [11, 12], whereas others show that hRSV SH protein of hRSV [13] and members of the family Paramyxoviridae (simian virus 5 - SV5 [14], parainfluenza virus 5 - PIV 5 [13], Mump virus - MuV) inhibit apoptosis in several mammalian cell lines.

Biochemical studies have shown that $\mathrm{SH}$ protein has a single hydrophobic region that spans the membrane, with a cytosolic tail at the carboxyl terminus [8]. Spectroscopic and NMR studies suggest SH secondary structure is composed of an $\alpha$-helix in the transmembrane domains (20-42) and a small $\alpha$-helix in the $C$ terminus [15]. In the presence of perfluorooctanoic acid (PFO) [16], in a BN-PAGE system and in a recent electron microscopy study on full-length SH protein in liposomes, $\mathrm{SH}$ protein has a compatible molecular weight and structure with five subunits in a pentameric arrangement [15]. Molecular modeling studies also suggest that SH can oligomerize as a pentameric protein and that the oligomer adopts a circular structure with a central pore at the $\alpha$-helix transmembrane domain with the hydrophobic side chains (Ile32, Ile36, Ile 40, and Leu44) facing the lumen of the channel domain [17]. In addition, SH increases membrane permeability to low-molecular-weight compounds in experiments of $\mathrm{SH}$ expression in E. coli [18]. Therefore, $\mathrm{SH}$ protein has been predicted to be a viroporin, a group of small highly hydrophobic viral proteins which can oligomerize and form pores in the cell membrane, thereby modifying membrane permeability to ions and small molecules [19]. In the face of the presented features and experimental insights, SH protein is a strong candidate for theoretical investigations regarding $\mathrm{hRSV}$ infection.

In this study, a combination of computational techniques, such as molecular modeling, molecular dynamics, and excited normal modes, allowed the proposal of a model for $\mathrm{SH}$ pentameric structure and dynamics, by the examination of the oligomer immersed in a model membrane surrounded by a solvent. A mechanism for the five SH subunits is suggested, which could explain the pathway for membrane permeability to small molecules, including water and ions, as well as its
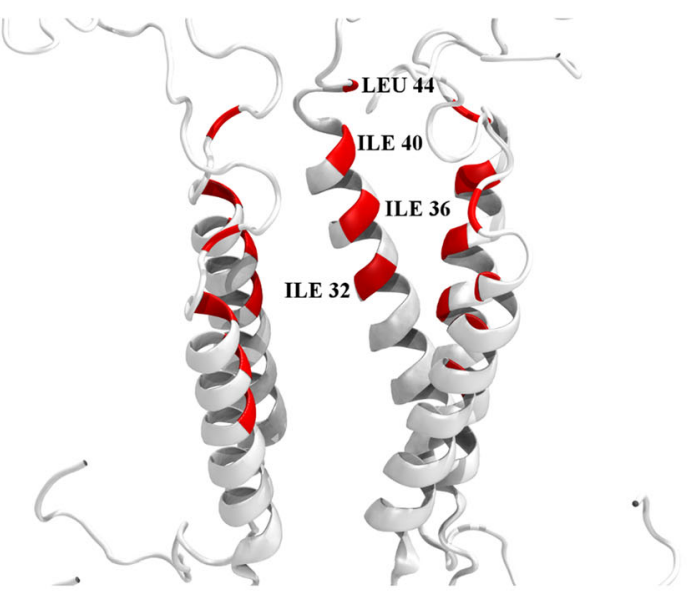

(A)

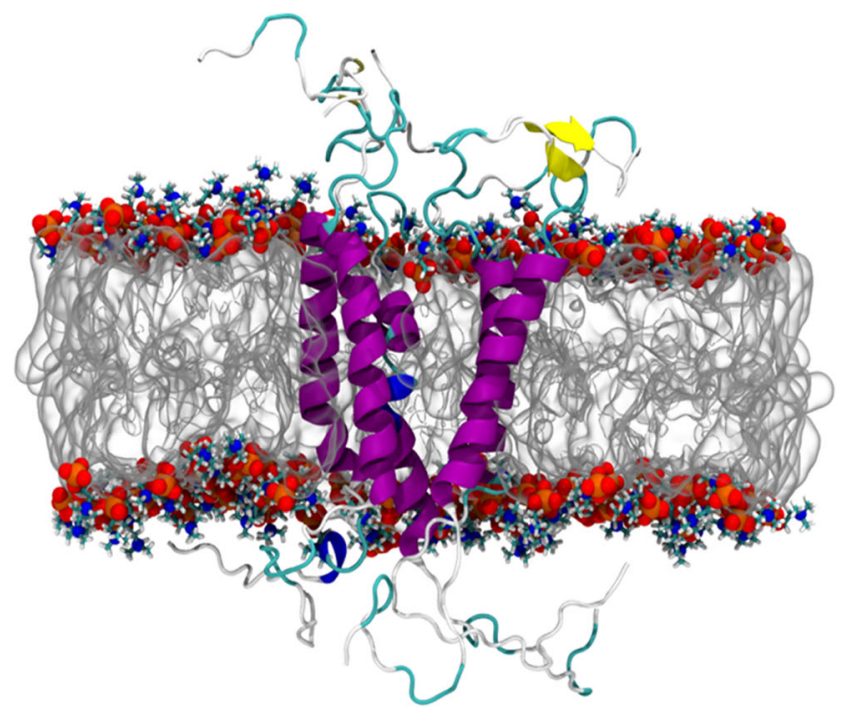

(B)

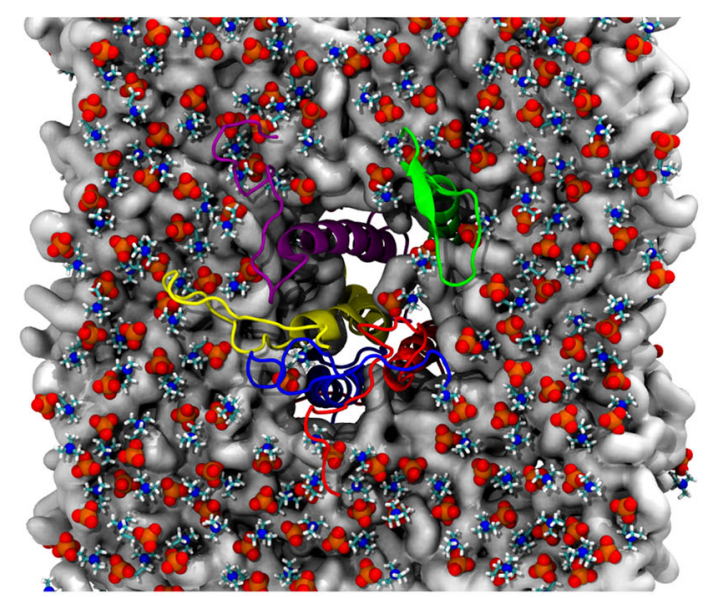

(C)

involvement in the delay of cellular apoptosis. SH simulations performed in the lipid bilayer allowed the observation of an 
Fig. 1 a Structural model of SH protein pentamer with the hydrophobic residues (Ile32, Ile36, Ile40, and Leu44) highlighted in red. b Side view and $\mathbf{c}$ top view snapshot of the SH pentameric model in POPC bilayer generated by the GROMACS tool g_cluster

increase in order parameter and area per lipid in the presence of the pentameric structure. In studies using molecular dynamics and excited normal modes, pore activity was observed due to the presence of water molecules inside the pore. The computational techniques employed in this work effectively provided specific details of atomic groups and key amino acids to SH dynamics. Hence, this study may serve as a microscope for experimentalists to understand how SH works, thus opening a potentially effective field for an antiviral therapy.

\section{Material and methods}

\section{SH protein modeling}

Since an SH model had not been proposed yet, protein modeling techniques were used to obtain a structural model. The analyses of secondary structure, hydrophobicity, and possible interaction sites of SH were performed using PREDICTPROTEIN [20] and PSIpred server [21]. The threading method was employed to predict SH structure due to low homology with other proteins with known experimental structure. The predicted SH 3D structure was constructed using I-TASSER server [22] based on ten templates. The chosen model was the one with a better estimated accuracy $(\mathrm{C}$-score -3.0 and TM-score $0.37 \pm 0.13)$ and in agreement with the experimental results, a TM domain with a single $\alpha$-helix [17]. Therefore, the values of C-score and TM-score and secondary structure in agreement with experimental results indicate a fairly good model and validate the SH model.

\section{Molecular dynamics simulation}

Molecular dynamics simulations were performed using GROMACS package (version 4.5.5) [23, 24]. GROMOS53A6 force field [25] was employed to model SH protein, Berger parametrization [26] to model lipids, and SPC as a water model [27]. Temperature was kept constant at $310 \mathrm{~K}$ by a V-rescale thermostat [28] with coupling constant $\tau=0.1 \mathrm{ps}^{-1}$. Constant pressure was held at $1 \mathrm{~atm}$ by a Berendsen barostat [29] with coupling constant $\tau=0.1 \mathrm{ps}^{-1}$. Integration of Newton's equation of motion was performed by the leapfrog algorithm with a time step of $2 \mathrm{fs}$. Long-range electrostatic interaction was treated using fast particle-mesh Ewald electrostatics (PME) [30]. A cutoff of $1.25 \mathrm{~nm}$ was implemented for Lennard-Jones and the direct space part of the Ewald sum for Coulombic interactions. The Fourier space part of Ewald splitting was computed by using the particle-mesh Ewald method [30], with a grid length of
$0.15 \mathrm{~nm}$. The overall positive electric charge of the system was neutralized by adding 1 ion of $\mathrm{Cl}^{-}$for each protein molecule.

Boxes of simulation with monomeric $\mathrm{SH}$ and pore pentameric structure in POPC bilayer were built using the following protocol. The orientation of the pore pentameric structure monomers in the membrane was based on an experimental and theoretical study proposed by Gan et al. [15] where the hydrophobic chains (Ile32, Ile36, Ile40, and Leu44) are oriented toward the pore lumen (Fig. 1a). The single pass helices were inserted in the same orientation. This structural feature is used to classify this protein as viroporin class I and subclass B (a single helix with a luminal amino terminus and cytosolic carboxyl terminus) [31]. Pore structure was minimized by the steep descent algorithm in order to rearrange side chains. SH monomer and pentamer were then superimposed in a solvated palmitoyl-oleoylphosphatidyl-choline (POPC) lipid bilayer patch consisting of 450 lipids (225 in each leaflet) and 88700 water molecules equilibrated at $1 \mathrm{~atm}$ and $310 \mathrm{~K}$ for $10 \mathrm{~ns}$, using full electrostatics and periodic boundary conditions. The area per lipid was calculated $\left(61.7 \pm 0.3 \AA^{2}\right)$ as a validation of the equilibration process performed in this study, which is in agreement with experimental values [32]. The membrane patch was sufficiently large $(12 \times 12 \mathrm{~nm})$ to accommodate the $\mathrm{SH}$ structures (either monomeric or pentameric). Most of the lipids overlapping with proteins were removed. Systems were minimized after monomeric/pentameric insertion by the steep descent algorithm and followed by MD simulation of $120 \mathrm{~ns}$ in which the last $50 \mathrm{~ns}$ were used for data acquisition.

\section{Molecular dynamics with excited normal modes (MDeNM)}

A recently developed method was applied, which combines normal modes analysis (NMA) and molecular dynamics (MD), called molecular dynamics with excited normal modes (MDeNM), in order to investigate the most significant movements of SH channel and the passage of water molecules through the protein $[33,34]$. NMA allows the investigation of conformational changes in proteins and other biomolecules [34]. The MDeNM method explores the normal mode and the Cartesian spaces [33]. In MDeNM simulations, several molecular dynamics simulations are performed in which the normal mode motions are kinetically activated [33]. This method was implemented via CHARMM script [35]. It is possible to use either only one or many linearly combined modes that are kinetically excited by inputting velocities along the modes. Finally, the major modes are selected to study the most relevant movements of interest for the present study.

The following computational protocol was used to investigate the conformational changes and movements of the target system: a) minimization (3000 steps) followed by equilibration ( $250 \mathrm{ps})$ and standard MD (100 ps) of the SH model using 
CHARMM [35], b) calculation of the first 62 lowestfrequency vibrational motions, c) analysis and selection of the modes that describe the most significant motions, d) molecular dynamics with excited normal modes (MDeNM) using all the linearly combined modes.

The SH pentamer (pore structure) was inserted in the homogenous bilayer membrane composed of dilauroylphosphatidyl-choline (DLPC) and a $20 \AA$ water thickness was included at the top and at the bottom of the protein inside the simulation box. A concentration of counter ions $\mathrm{Na}^{+}$and $\mathrm{Cl}^{-}$was included to neutralize the electrical charge of the system. Histidine 22 was protonated in all five chains of the pentamer. This procedure was performed using the CHARMM-GUI tool (www.charmm-gui.org), which creates the simulation input files. Five minimization steps were performed after including the membrane, water box and ions in order to equilibrate the system: a) four minimization steps using steep descent and ABNR algorithms with 25000 steps each, and b) one minimization step with steep descent and
ABNR with 50000 steps. Four short molecular dynamic runs were performed after minimization in order to equilibrate the system and one standard molecular dynamic production run with $100 \mathrm{ps}$ using canonic ensemble (NVT).

CHARMM package was used after the equilibration steps to calculate the normal modes of SH protein [34]. Lower normal mode frequencies represent the global conformational changes, whereas higher normal mode frequencies characterize local changes. The first 62 lowest frequency modes of the SH pentameric structure were calculated. Subsequently, the root mean square fluctuation (RMSF) was analyzed by residues. The 62 normal modes calculated were combined and used during the molecular dynamics with excited normal modes; 190 MDeNM replica runs were performed with ten excitations at the temperature $10 \mathrm{~K}$ [33].

It was observed by visual inspection of the $\mathrm{SH}$ chains end that chains $\mathrm{A}$ and $\mathrm{C}$, as well as chains $\mathrm{B}$ and $\mathrm{E}$, were diametrically opposed (Fig. 2). Thus, the opening and closing of one extremity was defined by the distances between $\alpha$-carbons of

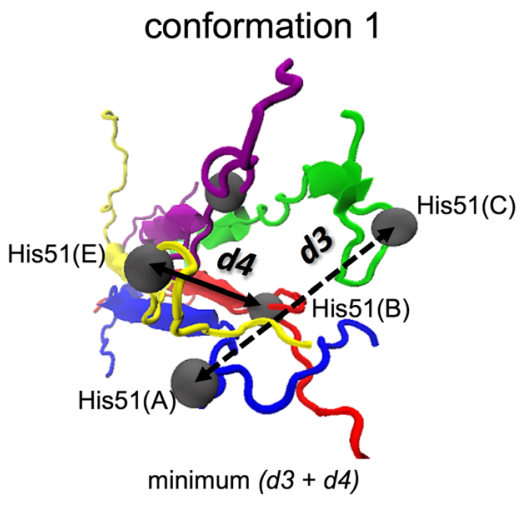

(A)

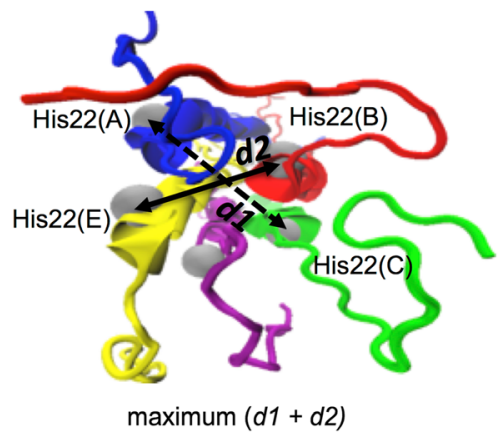

(D)

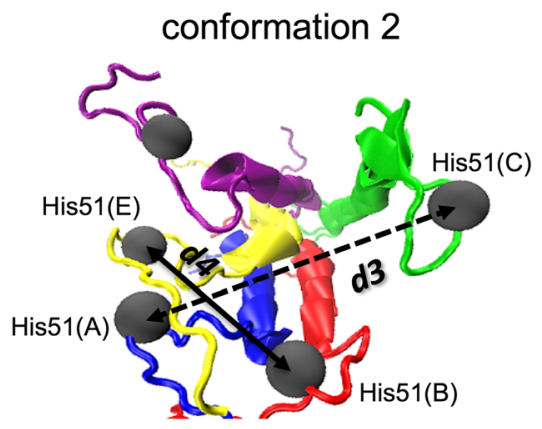

maximum $(d 3+d 4)$

(B)

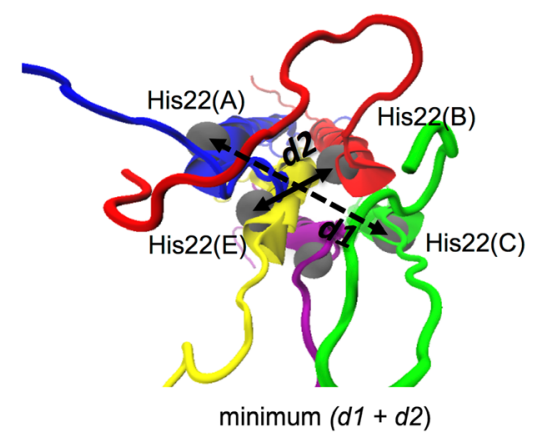

(E)

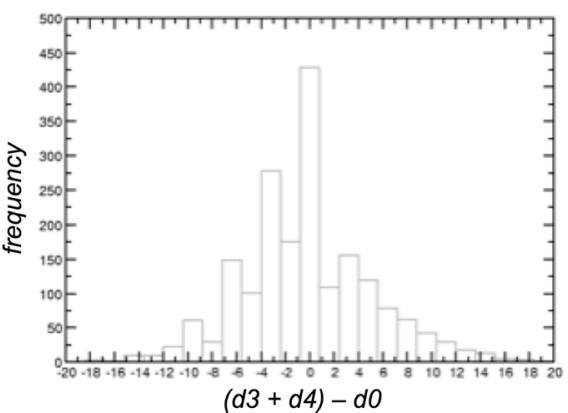

(C)

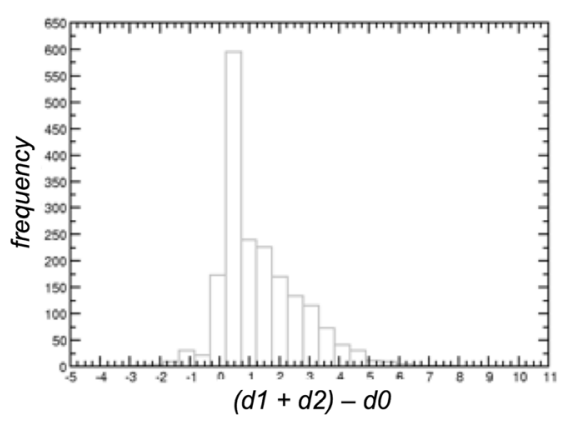

(F)
Fig. 2 The opening of the two extremities, conformations 1 (a) and d)) and 2 (b) and e)) of SH protein pentamer obtained by MDeNM. a Minimum and $\mathbf{b}$ maximum distances between histidines 51 at the $\mathrm{SH}$ structure. d Maximum and e minimum distances of histidines 22 at the pentamer protein. Chains A, B, C, D, and E are highlighted in blue, red, green, purple, and yellow, respectively. Distances were measured between histidine $\alpha$-carbons and defined as follows. $d 1$ is the distance between His 22 of chains A and C, $d 2$ is the distance between His 22 of chains $\mathrm{B}$ and $\mathrm{E}, d 3$ is the distance between His 51 of chains $\mathrm{A}$ and $\mathrm{C}, d 4$ is the distance between His 51 of chains B and E. Histidine $\alpha$-carbons are highlighted in gray in the structures. $\mathbf{c}$ Histogram of histidine 51 distances defined as $[(d 3+d 4)-d 0]$. $\mathbf{f}$ Histogram of histidine 22 distances defined as $[(d 1+d 2)-d 0] . d 0$ is the respective histidine distance of the reference structure with coordinates extracted after the MD energy minimization step, the starting point for the MDeNM production runs 
His51 of chains A and $\mathrm{C}(d 3)$ and His51 of chains B and E (d4) (Fig. 2a and b). The opening and closing of the other extremity was defined by the distances between $\alpha$-carbons of His 22 of chains A and $\mathrm{C}(d l)$ and His 22 of chains B and E (d2) (Fig. 2d and e). A script created in Visual Molecular Dynamics (VMD) [36] was employed to calculate all distances that were used to analyze the pore structure opening and closing distances in MDeNM replica runs.

\section{Results and discussion}

\section{SH protein and pentameric structure model}

SH monomeric and pentameric simulations in POPC were analyzed to determine their structural and dynamical properties. The analysis characterized the secondary structure stability of $\mathrm{SH}$ protein transmembrane domain (SH-TM) in a bilayer system. Figure 1 illustrates the SH pentameric model and the pore structure with a lipid bilayer membrane. The presence of $\alpha$-helix in the TM domain was in agreement with SH spectroscopy results [7, 14, 34] (Fig. 1). Changes in lipid order parameter were observed in the presence of an $\mathrm{SH}$ monomeric and pentameric protein. SH protein increases the order parameter of oleoyl and palmitoyl chains (Online resource), results also found in experiments on the effect of SH and SH-TM on lipid order [37, 38]. Decrease in the values of area per lipids were noticed from 61.7 $\pm 0.3 \AA^{2}$ to $57.4 \pm 0.3 \AA^{2}$, for a monomeric structure, and from $61.7 \pm 0.3 \AA^{2}$ to $48.8 \pm 0.3 \AA^{2}$, for a pentameric structure (Online Resource). These results suggest that the presence of SH protein in the bilayer positively contributes to membrane stability. Studies have reported that SH protein is involved in blocking host cell apoptosis by inhibiting TNF- $\alpha$ pathway [13, 39]. Moreover, these results suggest that the influence of SH protein on membrane fluidity is another possible inhibition mechanism of cell apoptosis, once membrane fluidity is an important physical property related to the induction of apoptosis [40, 41].

SH viroporin activity was analyzed by simulation of the pentamer inserted in a lipid bilayer. Once z-direction was considered normal to the plane of the membrane, distributions of molecular groups were obtained by slicing the box along this direction and by counting the number of molecules in each group in each slice. This produced a mean density profile as a function of $\mathrm{z}$. The resulting group distribution for water and several selected oleoyl chain carbon atoms are illustrated in the Online resource. Density distribution showed the presence of water molecules within the hydrophobic region of the bilayer, confirming that $\mathrm{SH}$ protein is a viroporin. The density profile is in agreement with the pore profile through the pentameric bundle of SH protein, an open funnel-shaped structure (Online resource).

The most likely SH pentameric model was chosen by cluster analysis performed with g_cluster GROMACS's tool [42]. Figure $1 \mathrm{~b}$ shows the pore conformation of $\mathrm{SH}$ protein pentamer inserted in the lipid bilayer with SH-TM domain composed by $\alpha$-helix. This model shows the pentameric structure anchored in the lipid bilayer with their $\mathrm{N}$-terminal intracellular-oriented and their C-terminal extracellular-oriented. These regions are composed by random coil and $\beta$-sheet structures, which can be an important strategy for pore functional mechanism to intermediate signal transduction between the intracellular and extracellular spaces. Studies suggest that SH protein may bind to BAP31 by the cytoplasmic domain, blocking the cleavage of BAP31 cytoplasmic vDED domain and avoiding the production of proapoptotic p20 [43]. This indicates the importance of knowledge of SH pore conformation to inhibit apoptosis.

\section{Normal modes analysis and molecular dynamics with excited normal modes (MDeNM)}

The 62 lowest normal modes were calculated using the program CHARMM as described by Philot et al. [34]. The first six modes are related to the translation and rotation of the system and were neglected. Mode 7 was the first mode with the lowest frequency (biggest amplitude in global conformational changes) to be analyzed and a torsional movement was observed by visual inspection. Mode 7 might be an important movement for pore permeability, as both extremities of SH pentamer rotate in opposite directions, opening the pore for small molecules.

The 62 modes were linearly combined for MDeNM simulations. We executed 190 replicas of MDeNM runs and measured the end openings of the SH pentameric structure given by distances defined between the representative $\alpha$-carbons of histidine residues at the chains. The structures in Fig. 2 represent two extremities among the conformations examined along the MDeNM replica runs: the most open end of the protein (Fig. 2a and d) and closed (Fig. 2b and e) conformations. Figure $2 \mathrm{a}$ and $\mathrm{b}$ shows the sum of His 51 distances $(d 3+$ $d 4$ ), which represents the open/close movement of one pore end. Figure $2 \mathrm{~d}$ and e presents the sum of His 22 distances $(d 1+d 2)$ with the close/open movement of the other pore end. A better description of the defined distances can be found in the Material and methods, Molecular dynamics with excited normal modes (MDeNM) section. All histidine conformation distances were counted and displayed as histograms populated as a function of $1 \AA$ bin (Fig. 2c and f). Figure 2c shows the histogram of His 51 distances obtained during the simulations, which were calculated as $[(d 3+d 4)-d 0]$, with $d 0$ being the His51 distances of the reference structure. Figure $2 \mathrm{f}$ presents the histogram of His 22 distances defined as $[(d 1+d 2)-d 0]$, with $d 0$ being the His 22 distance of the reference structure. The reference structure in Fig. $2 \mathrm{c}$ and $\mathrm{f}$ was generated with coordinates extracted after the MD energy minimization step, the starting point for the MDeNM production runs. It is worth noticing that in both histograms (Fig. 2c and f), near histidine 51, there is a wider opening (Fig. $2 \mathrm{a}$ and $\mathrm{b}$ ) than the other pore end (near histidine 22) (Fig. 2d and e). These results are in 
agreement with the pore water density and pore funnel-like profiles (Fig. $1 \mathrm{~b}$ and Online resource). The opening of both ends was significant, reaching values up to $18 \AA$ (Fig. 2c and $\mathrm{f}$ ), which is corroborated by the experimental value of $19 \AA$ for the SH pentamer pore core width [44]. This opening of pore ends was enough to allow water molecules and ions to cross from the intracellular to extracellular medium through its structure (Fig. 3 and Online resource), changing the normal cellular function. Several structures obtained in the replicas by MDeNM showed water molecules and ions inside the pore, which can be seen in one representative structure in Fig. 3.

Studies suggest that ion channel activity through $\mathrm{SH}$ protein may contribute to the production of IL- $1 \beta$ by the activation of the inflammasome [45]. This induction is inhibited by a treatment with HMA and $\mathrm{Na}^{+}-\mathrm{H}^{+}$ion channel inhibitor [46], proposing that viral viroporins ion channel activity disrupts the intracellular ion balance [47]. In addition, ion channels may control apoptosis in cells $[48,49]$. The virus will have advantages in its replication if SH protein inhibits the apoptosis in host cells during infection, since the infected cell will survive longer, thus resulting in more viral replications. This mechanism can contribute to increase virulence factor during infection. Recent studies have shown a high dependence of ion channel activity relating to residues His22 and His51 [36]. Our results show that these residues play a key role in the opening and closing mechanism of the pentameric structure, since they are located in strategic places within the chains, near N- and C-terminals (Fig. 2). These histidines might act as a selective mechanism for the pore.

The MDeNM methodology, a combination of normal modes analysis with molecular dynamics, allowed the exploration of hRSV SH pore conformational spaces very efficiently. This is a successful indication that the MDeNM protocol employed in this work can be used to study transmembrane

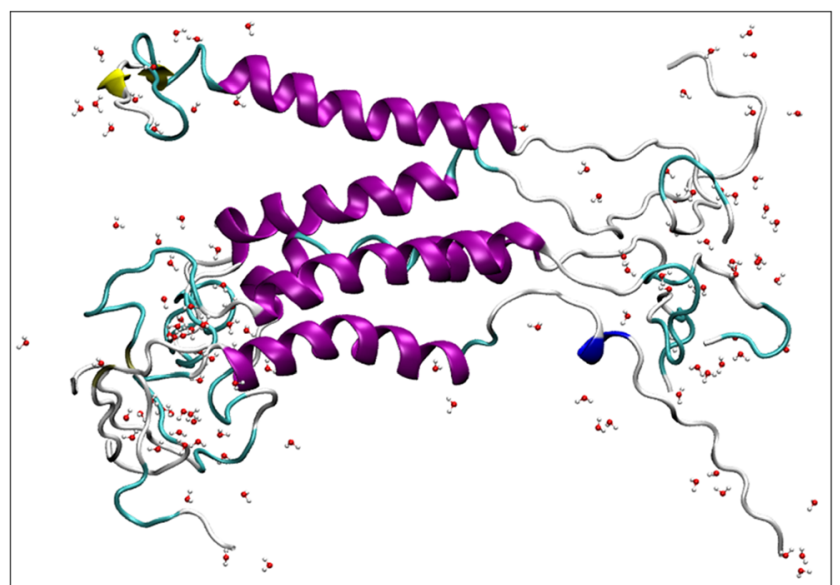

Fig. 3 Representation of pore structure with water molecules inside. Representative structure extracted from one of the MDeNM replicas, which shows the passage of water molecules through the $\mathrm{SH}$ pentameric pore proteins with long time scale dynamics, which has a high computational cost to be performed by pure MD simulations.

\section{Conclusions}

SH protein has been predicted to be a viroporin. In this study, we presented a structural model for SH protein and its pore conformation developed with molecular modeling, molecular dynamics simulations, and an improved version of normal mode analysis. The pentameric model, investigated in this study, was suggested by experimental data from the literature [50]. The pentameric model was stable inside the lipid bilayer during the entire molecular dynamics simulation, suggesting that the presence of a pentameric structure increases membrane stability. This feature can be a possible mechanism involved in the inhibition of cellular apoptosis and, consequently, in the success of viral infectivity when $\mathrm{SH}$ protein gene is present.

The most likely SH pentameric model shows the pentameric structure anchored in the lipid bilayer with their $\mathrm{N}$-terminal and their $\mathrm{C}$-terminal composed by random coil and $\beta$-sheet structures. These regions are important for interaction with viral and host proteins and can be important to inhibit ligand-mediated apoptosis in cells.

The results of modified normal modes analysis (MDeNM) showed functional movements that open one end of the pore and close the other, allowing a permeability of small molecules through the channel. These movements suggest an important mechanism for viroporin activity. MDeNM simulations generated several structures where water molecules overpass the pore. The combination of normal modes excited by molecular dynamics was efficient to investigate functional conformation changes in transmembrane proteins that act as small molecule channels.

Our results reinforce the key role of SH protein in the cellular homeostasis control during viral infection and in interactions with viral and host proteins. This viral strategy can be used to keep the cell alive longer, promoting higher viral replication rates. This theoretical study can help the investigation of drug candidates to inhibit SH protein, since the inhibition of viroporin activity or protein-protein interactions would lead to a low production of viruses. This might be enough for the immune system to eradicate the viral infection.

Acknowledgments The authors would like to thank the Brazilian Agencies FAPEMIG, CAPES, and CNPq for the financial support and the facilities GridUNESP, CENAPAD-SP, and V.B.P. Leite's cluster (FAPESP grant 2011/17658-3) for the computational resources. ASA was supported by FAPESP grant 2010/18169-3.

\section{Compliance with ethical standards}

Competing interests The authors declare they have no competing interests. 


\section{References}

1. Falsey AR, Hennessey PA, Formica MA, Cox C, Walsh EE (2005) Respiratory syncytial virus infection in elderly and high-risk adults. N Engl J Med 352:1749-1759. doi:10.1056/NEJMoa043951

2. Nair H, Nokes DJ, Gessner BD, Dherani M, Madhi SA, Singleton RJ et al. (2010) Global burden of acute lower respiratory infections due to respiratory syncytial virus in young children: a systematic review and meta-analysis. Lancet 375: 1545-1555. doi:10.1016/S0140-6736(10)60206-1

3. Boyce TG, Mellen BG, Mitchel EF, Wright PF, Griffin MR (2000) Rates of hospitalization for respiratory syncytial virus infection among children in medicaid. J Pediatr 137:865-870. doi: $10.1067 / \mathrm{mpd} .2000 .110531$

4. Collins PL, Hill MG, Cristina J, Grosfeld H (1996) Transcription elongation factor of respiratory syncytial virus, a nonsegmented negative-strand RNA virus. Proc Natl Acad Sci 93:81-85

5. Kochva U, Leonov H, Arkin IT (2003) Modeling the structure of the respiratory syncytial virus small hydrophobic protein by silentmutation analysis of global searching molecular dynamics. Protein Sci Publ Protein Soc 12:2668-2674

6. Collins PL, Olmsted RA, Johnson PR (1990) The small hydrophobic protein of human respiratory syncytial virus: comparison between antigenic subgroups A and B. J Gen Virol 71(Pt 7):1571-1576

7. Olmsted RA, Collins PL (1989) The 1A protein of respiratory syncytial virus is an integral membrane protein present as multiple, structurally distinct species. J Virol 63:2019-2029

8. Collins PL, Mottet G (1993) Membrane orientation and oligomerization of the small hydrophobic protein of human respiratory syncytial virus. J Gen Virol 74(Pt 7):1445-1450

9. Jin H, Zhou H, Cheng X, Tang R, Munoz M, Nguyen N (2000) Recombinant respiratory syncytial viruses with deletions in the $\mathrm{NS} 1, \mathrm{NS} 2, \mathrm{SH}$, and M2-2 genes are attenuated in vitro and in vivo. Virology 273:210-218. doi:10.1006/viro.2000.0393

10. Whitehead SS, Bukreyev A, Teng MN, Firestone CY, Claire MS, Elkins WR et al. (1999) Recombinant respiratory syncytial virus bearing a deletion of either the NS2 or SH gene is attenuated in chimpanzees. J Virol 73:3438-3442

11. Heminway BR, Yu Y, Tanaka Y, Perrine KG, Gustafson E, Bernstein JM et al. (1994) Analysis of respiratory syncytial virus F, G, and SH proteins in cell fusion. Virology 200:801-805. doi:10.1006/viro.1994.1245

12. Techaarpornkul S, Barretto N, Peeples ME (2001) Functional analysis of recombinant respiratory syncytial virus deletion mutants lacking the small hydrophobic and/or attachment glycoprotein gene. J Virol 75:6825-6834. doi:10.1128/JVI.75.15.68256834.2001

13. Fuentes S, Tran KC, Luthra P, Teng MN, He B (2007) Function of the respiratory syncytial virus small hydrophobic protein. J Virol 81:8361-8366. doi:10.1128/JVI.02717-06

14. He B, Lin GY, Durbin JE, Durbin RK, Lamb RA (2001) The SH integral membrane protein of the paramyxovirus simian virus 5 is required to block apoptosis in MDBK cells. J Virol 75:4068-4079. doi:10.1128/JVI.75.9.4068-4079.2001

15. Gan S-W, Tan E, Lin X, Yu D, Wang J, Tan GM-Y et al. (2012) The small hydrophobic protein of the human respiratory syncytial virus forms pentameric ion channels. J Biol Chem 287:24671-24689. doi:10.1074/jbc.M111.332791

16. Ramjeesingh M, Huan LJ, Garami E, Bear CE (1999) Novel method for evaluation of the oligomeric structure of membrane proteins. Biochem J 342:119-123

17. Gan SW, Ng L, Lin X, Gong X, Torres J (2008) Structure and ion channel activity of the human respiratory syncytial virus (hRSV) small hydrophobic protein transmembrane domain. Protein Sci Publ Protein Soc 17:813-820. doi:10.1110/ps.073366208
18. Perez M, Garcia-Barreno B, Melero JA, Carrasco L, Guinea R (1997) Membrane permeability changes induced in escherichia coliby the sh protein of human respiratory syncytial virus. Virology 235:342-351. doi:10.1006/viro.1997.8696

19. Gonzalez ME, Carrasco L (2003) Viroporins. FEBS Lett 552:28-34

20. Rost B, Yachdav G, Liu J (2004) The PredictProtein server. Nucleic Acids Res 32:W321-W326. doi:10.1093/nar/gkh377

21. Buchan DWA, Minneci F, Nugent TCO, Bryson K, Jones DT (2013) Scalable web services for the PSIPRED protein analysis workbench. Nucleic Acids Res 41:W349-W357. doi:10.1093/nar/gkt381

22. Zhang Y (2008) I-TASSER server for protein 3D structure prediction. BMC Bioinf 9:40. doi:10.1186/1471-2105-9-40

23. Berendsen HJC, van der Spoel D, van Drunen R (1995) GROMACS: a message-passing parallel molecular dynamics implementation. Comput Phys Commun 91:43-56. doi:10.1016/0010-4655(95)00042-E

24. Pronk S, Páll S, Schulz R, Larsson P, Bjelkmar P, Apostolov R et al. (2013) GROMACS 4.5: a high-throughput and highly parallel open source molecular simulation toolkit. Bioinformatics doi:10.1093/ bioinformatics/btt055.

25. Oostenbrink C, Villa A, Mark AE, van Gunsteren WF (2004) A biomolecular force field based on the free enthalpy of hydration and solvation: the GROMOS force-field parameter sets 53A5 and 53A6. J Comput Chem 25:1656-1676. doi:10.1002/jcc.20090

26. Berger O, Edholm O, Jähnig F (1997) Molecular dynamics simulations of a fluid bilayer of dipalmitoylphosphatidylcholine at full hydration, constant pressure, and constant temperature. Biophys J 72:2002-2013. doi:10.1016/S0006-3495(97)78845-3

27. Berendsen HJC, Postma JPM, van Gunsteren WF, Hermans J (1981) Interaction models for water in relation to protein hydration. In: Pullman B (ed) Intermolecular forces. Springer, Netherlands, pp 331-342

28. Bussi G, Donadio D, Parrinello M (2007) Canonical sampling through velocity rescaling. J Chem Phys 126:014101. doi:10.1063/1.2408420

29. Berendsen HJC, Postma JPM, van Gunsteren WF, DiNola A, Haak JR (1984) Molecular dynamics with coupling to an external bath. J Chem Phys 81:3684-3690. doi:10.1063/1.448118

30. Essmann U, Perera L, Berkowitz ML, Darden T, Lee H, Pedersen LG (1995) A smooth particle mesh Ewald method. J Chem Phys 103:8577-8593. doi:10.1063/1.470117

31. Nieva JL, Madan V, Carrasco L (2012) Viroporins: structure and biological functions. Nat Rev Microbiol 10:563-574. doi: $10.1038 /$ nrmicro2820

32. Murzyn K, Róg T, Pasenkiewicz-Gierula M (2005) Phosphatidylethanolamine-phosphatidylglycerol bilayer as a model of the inner bacterial membrane. Biophys J 88:1091-1103. doi:10.1529/biophysj.104.048835

33. Costa MGS, Batista PR, Bisch PM, Perahia D (2015) Exploring free energy landscapes of large conformational changes: molecular dynamics with excited normal modes. J Chem Theory Comput. doi:10.1021/acs.jctc.5b00003

34. Floquet N, Durand P, Maigret B, Badet B, Badet-Denisot M-A, Perahia D (2009) Collective motions in glucosamine-6-phosphate synthase: influence of ligand binding and role in ammonia channelling and opening of the fructose-6-phosphate binding site. J Mol Biol 385:653-664. doi:10.1016/j.jmb.2008.10.032

35. Brooks BR, Bruccoleri RE, Olafson BD, States DJ, Swaminathan S, Karplus M (1983) CHARMM: a program for macromolecular energy, minimization, and dynamics calculations. J Comput Chem 4:187-217. doi:10.1002/jcc.540040211

36. Li Y, To J, Verdià-Baguena C, Dossena S, Surya W, Huang M et al. (2014) Inhibition of the human respiratory syncytial virus small hydrophobic protein and structural variations in a Bicelle environment. J Virol 88:11899-11914. doi:10.1128/JVI.00839-14 
37. Wan S, Torres J (2011) Structural and functional aspects of the small hydrophobic $(\mathrm{SH})$ protein in the human respiratory syncytial virus. In: Resch B (ed) Hum Respir Syncytial Virus Infect. InTech, doi: $10.5772 / 28747$

38. Tamm LK, Tatulian SA (1997) Infrared spectroscopy of proteins and peptides in lipid bilayers. Q Rev Biophys 30:365-429

39. Lin Y, Bright AC, Rothermel TA, He B (2003) Induction of apoptosis by paramyxovirus simian virus 5 lacking a small hydrophobic gene. J Virol 77:3371-3383. doi:10.1128/JVI.77.6.33713383.2003

40. Jourd'heuil D, Aspinall A, Reynolds JD, Meddings JB (1996) Membrane fluidity increases during apoptosis of sheep ileal Peyer's patch B cells. Can J Physiol Pharmacol 74:706-711

41. Fujimoto K, Iwasaki C, Kawaguchi H, Yasugi E, Oshima M (1999) Cell membrane dynamics and the induction of apoptosis by lipid compounds. FEBS Lett 446:113-116. doi:10.1016/S0014-5793(99)00204-5

42. Wilson RL, Fuentes SM, Wang P, Taddeo EC, Klatt A, Henderson AJ et al. (2006) Function of small hydrophobic proteins of paramyxovirus. J Virol 80:1700-1709. doi:10.1128/JVI.80.4.17001709.2006

43. Triantafilou K, Kar S, Vakakis E, Kotecha S, Triantafilou M (2013) Human respiratory syncytial virus viroporin $\mathrm{SH}$ : a viral recognition pathway used by the host to signal inflammasome activation. Thorax 68:66-75. doi:10.1136/thoraxjnl-2012-202182
44. Chen I-Y, Ichinohe T (2015) Response of host inflammasomes to viral infection. Trends Microbiol 23:55-63. doi:10.1016/j. tim.2014.09.007

45. Daura X, Gademann K, Jaun B, Seebach D, van Gunsteren WF, Mark AE (1999) Peptide folding: when simulation meets experiment. Angew Chem Int Ed 38:236-240. doi:10.1002/(SICI)15213773(19990115)38:1/2<236::AID-ANIE236>3.0.CO;2-M

46. Li Y, Jain N, Limpanawat S, To J, Quistgaard EM, Nordlund P et al. (2015) Interaction between human BAP31 and respiratory syncytial virus small hydrophobic (SH) protein. Virology 482:105-110. doi:10.1016/j.virol.2015.03.034

47. Ambroggio EE, Separovic F, Bowie JH, Fidelio GD, Bagatolli LA (2005) Direct visualization of membrane leakage induced by the antibiotic peptides: maculatin, citropin, and aurein. Biophys J 89: 1874-1881. doi:10.1529/biophysj.105.066589

48. Lang F, Föller M, Lang KS, Lang PA, Ritter M, Gulbins E et al. (2005) Ion channels in cell proliferation and apoptotic cell death. J Membr Biol 205:147-157. doi:10.1007/s00232-005-0780-5

49. Szabò I, Adams C, Gulbins E (2004) Ion channels and membrane rafts in apoptosis. Pflügers Arch Eur J Physiol 448:304-312

50. Kochva U, Leonov H, Arkin IT (2003) Modeling the structure of the respiratory syncytial virus small hydrophobic protein by silentmutation analysis of global searching molecular dynamics. Protein Sci 12:2668-2674. doi:10.1110/ps.03151103 